Brown Dwarfs and Extrasolar Planets

ASP Conference Series, Vol. 134, 1998

R. Rebolo, E. L. Martín and M. R. Zapatero Osorio (eds.)

\title{
A New Population of Brown Dwarfs
}

\author{
M.R.S. Hawkins \\ Royal Observatory, Blackford Hill, Edinburgh EH9 зHJ, UK \\ C. Ducourant \\ Observatoire de Bordeaux, URA 352, CNRS/INSU, B.P. 89, F-33270 \\ Floirac, France \\ H.R.A. Jones \\ Astronomy Group, LMU, Liverpool L3 3AF, UK \\ Institute of Astronomy, University of Tokyo, Mitaka 181, Japan \\ M. Rapaport \\ Observatoire de Bordeaux, URA 352, CNRS/INSU, B.P. 89, F-33270 \\ Floirac, France
}

\begin{abstract}
A large area deep survey for brown dwarfs from digitally stacked $1.2 \mathrm{~m}$ Schmidt plates has revealed a population of very red stars (R-I > 3). On the basis of their colours, near IR spectra and their luminosity estimates from proper motions, these objects appeared to be Brown Dwarfs. First measurements of the parallaxes of 8 of the reddest objects at the 1.5 danish telescope confirmed this, and showed that four of them have K-band luminosities some mags below the bottom of the main sequence. We present here those results.
\end{abstract}

\section{Schmidt Survey: the survey for low mass stars}

The survey for low mass stars was carried out using Schmidt plates from the UK Schmidt telescope (Siding Spring, Australia), including $65 \mathrm{R}$ and $30 \mathrm{I}$ band plates. These plates were digitised by the COSMOS measuring machine, and digitally stacked providing a catalog of 285000 objects.

Proper motions : Proper motions for all stars were evaluated by comparing the I first epoch positions with the I late epoch positions (a base of time of 10 years).

Selection of low mass stars: Stars were selected when R-I $>3.0$

\section{Spectroscopy}

The observations were made with the FORS spectrograph on the Anglo-Australian Telescope. The spectra showed a number of features indicating very low tem- 
perature including exceptionally strong $\mathrm{TiO}$ and VO features, and extensive absorption due to $\mathrm{FeH}$. Frequently the region around $740 \mathrm{~nm}$ tends to be enhanced compared to the shorter wavelenght region for a given spectral type measured from other features. This explains why their R-I colours are so red.

\section{Infrared photometry}

Observations were held at the ESO $2.2 \mathrm{~m}$ at La Silla with the infrared camera IRAC2. In the derived optical and IR colour/colour diagrams constituted with a selection of the reddest stars in our sample together with some standard stars we see that the standards form a well defined locus, the stars from our sample are broadly scattered across the diagram with no clear correlation between the various colour. The apparently contradictory indicators of the nature of our sample could only be resolved with direct measures of luminosity which is to say that we need the trigonometric parallaxes.

\section{Trigonometric parallaxes}

Observations of 5 brown dwarf candidates were carried out at three epochs $(5 / 94$, 11/94, 6/95) at the ESO $1.54 \mathrm{~m}$ Danish Telescope equiped with a 1024x1024 pixels CCD camera. Frames were measured using DAOPHOT routine fitting a PSF constructed for each frame. Measurements were corrected from DCR effects when a correction could be calculated. Then the frames were cross-identified to a master frame and astrometrically reduced as described in Ducourant et al (this volume).

The resolution of the system is done using a Gauss-Seidel type iterative method. A constraint is added (non significant parallaxes are forced to be nul) in order to treat a non-singular system. Results are presented in Hawkins et al(1997).

\section{Results}

Of the 5 target stars, the two faintest did not have significant parallaxes. The remainder have distances in the range 23 to 48 parsecs with an error of about 20 percent. For comparison purpose we also included in our program a brighter very red dwarf, with two independently measured parallaxes (Ianna 1992, Tinney 1996). Our result is within $1.5 \mathrm{~s}$ from Ianna's value and 1s from Tinney's one. This excellent agreement give us good confidence that the other target parallaxes are reliable.

Absolute magnitudes were derived from those measurements and are presented in Hawkins et al (1997). The new objects inhabit a hitherto unoccupied part of the H-R diagram. They have similar colours to M6-7 objects though they are around three magnitudes fainter. Their low absolute $\mathrm{K}$ magnitudes put them in a regime substantially below the current model predictions for the faintest objects which can burn hydrogen for any metallicity.

On the basis of their position in this diagram one can deduce that the new stars are metal poor with an age around 5 Gyr. This would imply a mass of 
about 0.06 solar masses. It is still too early to answer the important question of the space density of the stars. However, if we conservatively assume that their mass is around 0.06 solar masses and make realistic estimates of the completeness of the sample, the three stars so far discovered represent a space density which appears to be consistent with a mass function rising steadily into the brown dwarf regime.

\section{References}

Ianna P.A., in Developments in Astrometry and their Recent Impacts on Astrophysics and Geodynamics (ed Mueller, Kolaczek) IAU Symp. 156, p.75

Tinney C.G., 1993, AJ, 105, 1169

Tinney C.G., 1996, MNRAS, 281, 644

Stetson P.B., 1987, PASP, 99, 191 